\title{
Selección óptima de conductores en sistemas de distribución empleando el algoritmo búsqueda tabú
}

\author{
Optimal selection of conductors in distribution systems \\ using tabu search algorithm
}

\author{
O.D. Montoya G. ${ }^{*}$ \\ A. Grajales ${ }^{2}$ \\ R.A. Hincapié I. ${ }^{1}$ \\ Recibido 30 de junio de 2016, Aceptado 27 de marzo de 2017 \\ Received: June 30, 2016 Accepted: March, 27, 2017.
}

\begin{abstract}
RESUMEN
En este trabajo se presenta una metodología eficiente para la selección óptima de conductores en sistemas de distribución (SD) por medio de la implementación de la técnica de optimización metaheurística búsqueda tabú (BT). Para representar el problema es empleado un modelo matemático monoobjetivo del tipo no lineal entero mixto. Como función objetivo, es considerada la minimización de los costos de inversión en conductores en conjunto con las pérdidas técnicas de la red para un período de estudio de un año, considerando un equivalente de red monofásico. El conjunto de restricciones corresponde a las características técnicas y operativas del sistema, las que son evaluadas por medio de un flujo de potencia del tipo barrido iterativo. Para construir la configuración inicial para el BT, es empleado un algoritmo heurístico constructivo que garantiza factibilidad de la solución inicial y para la generación del vecindario, son utilizadas estrategias de búsqueda en árbol y teoría de grafos. Con el fin de verificar aplicabilidad y eficiencia, se emplearon dos sistemas de la literatura especializada, obteniendo resultados de mejor calidad que los reportados en la literatura especializada.
\end{abstract}

Palabras clave: Algoritmos constructivos, búsqueda tabú, dimensionamiento de conductores, optimización monoobjetivo, sistemas de distribución.

\begin{abstract}
An efficient methodology for the optimal selection of conductor in distribution systems through the implementation of the metaheuristic optimization technique called Tabu Search is presented in this paper. To represent the problem a mono-objective mathematical model of nonlinear mixed integer type is used. The minimization of investment costs of conductors and operating costs (power losses) for a study period of one year is considered as objective function. A single-phase equivalent system is used for the evaluation. The set of constraints relates to technical and operational characteristics of the system, which are assessed through a backward/forward sweep load flow. Initial configuration to tabu search algorithm is created by using a constructive heuristic that ensures feasibility of the initial solution; to generate the neighborhood strategies of tree searching and graph theory are used. In order to verify its applicability and efficiency, two test feeders of specialized literature are employed, obtaining better quality results than those reported in the literature.
\end{abstract}

Keywords: Constructive algorithm, tabu search, conductors sizing, mono-objective optimization, distribution systems.

\footnotetext{
1 Programa de Ingeniería Eléctrica e Ingeniería Electrónica, Facultad de Ingeniería, Universidad Tecnológica de Bolívar, Km 1 vía Turbaco, Cartagena, Colombia. E-mail: omontoya@utb.edu.co

2 Programa de Ingeniería Eléctrica. Facultad de Ingenierías. Universidad Tecnológica de Pereira. Carrera 27 \#10-02 Barrio Álamos Pereira, Colombia..E-mail: agrajales87@gmail.com; ricardohincapie@utp.edu.co

* Autor de correspondencia.
} 


\section{NOMENCLATURA}

\section{Conjuntos}

$\Omega_{c} \quad$ Conjunto que contiene todos los tipos de conductores disponibles.

$\Omega_{L} \quad$ Conjunto que contiene todos los tramos de red del SD.

$\Omega_{H} \quad$ Conjunto que contiene los períodos de duración de carga.

$\Omega_{N} \quad$ Conjunto que contiene todos los nodos del SD.

$S \quad$ Espacio de soluciones factibles asociadas a un problema de optimización.

\section{Parámetros, subíndices y superíndices}

$b_{i j}^{c} \quad$ Suceptancia asociada al tramo de red $i j$ con calibre tipo $c[\mathrm{~km} / \Omega]$.

C Superíndice asociado al tipo de calibres de conductores disponibles.

CIC $^{c}$ Costo de instalación por kilómetro del conductor con calibre tipo $c$ [US\$/km].

$C_{W h} \quad$ Costo de la energía [US\$/Wh].

$D C C_{h} \quad$ Duración de la curva de demanda para el período $h[\mathrm{~h}]$.

$g_{i j}^{c} \quad$ Conductancia asociada al tramo de red $i j$ con calibre tipo $c[\mathrm{~km} / \Omega]$.

$h \quad$ Subíndice asociado a los intervalos de la curva de carga discretizada.

$i, i j, k l \quad$ Subíndices asociados a los nodos y los tramos de red del SD, respectivamente.

$I_{i j}^{c, \max }$ Capacidad térmica de conducción de corriente para el conductor $c$ con calibre tipo $c$ asociado al tramo de red $i j[\mathrm{~A}]$.

$L_{i j} \quad$ Longitud del tramo de red $i j[\mathrm{~km}]$.

$P_{i, h}^{D} \quad$ Potencia activa demandada en el nodo $i$ en el período $h$ [W].

$Q_{i, h}^{D} \quad$ Potencia reactiva demandada en el nodo $i$ en el período $h$ [VAr].

$r_{i j}^{c} \quad$ Resistencia del conductor con calibre tipo $c$ conectado entre los nodos $i$ y $j[\Omega / \mathrm{km}]$.

$V_{i}^{\max } \quad$ Límite máximo de regulación de tensión en el nodo $i[\mathrm{~V}]$.

$V_{i}^{\min } \quad$ Límite mínimo de regulación de tensión en el nodo $i[\mathrm{~V}]$.

$T_{i j, k l} \quad$ Matriz que indica si el tramo de red $i j$ es la línea inmediatamente aguas arriba del tramo de red $k l$.

$x_{i i}^{c} \quad$ Reactancia inductiva del conductor con calibre tipo $c$ conectado entre los nodos $i$ y $j[\Omega / \mathrm{km}]$.

$\alpha_{c} \quad$ Parámetro que indica el calibre asociado al conductor tipo $c$.

\section{Variables}

$I_{i j . h}^{c} \quad$ Magnitud de la corriente que circula por el tramo de red $i j$ con calibre tipo $c$ en el período $h[\mathrm{~A}]$.

$I_{i j, h}^{i, c} \quad$ Componente imaginaria de la corriente que circula por el tramo de red $i j$ con calibre tipo $c$ en el período $h$ [A].

$I_{i j, h}^{r, c} \quad$ Componente real de la corriente que circula por el tramo de red $i j$ con calibre tipo $c$ en el período $h[\mathrm{~A}]$.

$P_{i, h}^{G} \quad$ Potencia activa generada en el nodo $i$ en el período $h$ [W].

$P_{i j, h}^{c} \quad$ Flujo de potencia activa por el tramo de red $i j$ con calibre tipo $c$ en el período $h$ [W].

$Q_{i, h}^{G} \quad$ Potencia reactiva generada en el nodo $i$ en el período $h$ [VAr].

$Q_{i j, h}^{c} \quad$ Flujo de potencia reactiva por el tramo de red $i j$ con calibre tipo $c$ en el período $h$ [W].

$V_{i, h} \quad$ Magnitud de la tensión en el nodo $i$ en el período $h[\mathrm{~V}]$.

$V_{j, h} \quad$ Magnitud de la tensión en el nodo $j$ en el período $h[\mathrm{~V}]$.

$x \quad$ Variables de decisión de un problema genérico de optimización,

$\delta_{i j}^{c}, \delta_{k l}^{c} \quad$ Variable de decisión que indica si un conductor con calibre tipo $c$ es instalado en el tramo de red $i j(k l)$. (valor de 1 indica que si se instala, valor de 0 , que no se instala).

$\theta_{i, h} \quad$ Ángulo de la tensión en el nodo $i$ en el período $h$ [rad].

$\theta_{j, h} \quad$ Ángulo de la tensión en el nodo $j$ en el período $h$ [rad].

$\theta_{i j, h} \quad$ Diferencia angular entre las tensiones de los nodos $i$ y $j$ en el período $h$ [rad].

\section{Funciones}

$f(x) \quad$ Función genérica de un problema de optimización correspondiente a la función objetivo.

$\mathrm{g}(x) \quad$ Función genérica de un problema de optimización que corresponde al conjunto de restricciones de desigualdad.

$h(x) \quad$ Función genérica de un problema de optimización que corresponde al conjunto de restricciones de igualdad.

$\tilde{z} \quad$ Función objetivo con penalizaciones [US\$]. Función objetivo [US\$].

máx $(\cdot) \quad$ Función que maximiza/minimiza la cantidad 
mín( $\cdot) \quad$ la función contenida en el paréntesis. máx\{a, Función que calcula el máximo o el mín $\{a, 1$ mínimo de las cantidades contenidas entre las llaves, es decir, si $a$ es mayor que $b$, entonces $\operatorname{máx}\{a, b\}=a$ y $\min \{a, b\}=b$.

\section{INTRODUCCIÓN}

El creciente aumento de la demanda de energía eléctrica impulsada por el constante crecimiento de las ciudades y la expansión de las capacidades productivas del sector industrial, generan una inevitable expansión del sistema eléctrico para poder atender de manera oportuna, eficiente y confiable toda la demanda requerida [1].

Bajo este escenario, las compañías de distribución (DisCo) están en la necesidad de expandir sus sistemas eléctricos en el corto y mediano plazo, de forma que se obtengan los menores costos en inversión y operación para el período de tiempo considerado en el horizonte de estudio; la expansión del sistema puede ser proyectada de manera estática o dinámica y esto depende de los criterios que cada DisCo aplique en sus proyectos de expansión [2].

En los procesos de planeación de la expansión de SD, siempre son considerados como objeto de minimización, los costos de inversión en nuevas subestaciones y tramos de red, así como su aumento de capacidad, además de los costos asociados a las pérdidas [1-3]. Dentro de este problema existe un subproblema relacionado con la selección óptima de los conductores que serán instalados en la red, ya que esto impacta directamente los costos de inversión y operación [4].

La selección de los mejores conductores para las redes de distribución depende claramente de las consignas operativas de la DisCo; sin embargo, en términos generales, este es susceptible de ser aplicado para redes nuevas y existentes. Para las redes nuevas, se seleccionan los conductores dentro de un grupo de candidatos disponibles (diferentes calibres) siendo ya conocidas las rutas de los alimentadores (topología fija) [5]. Para las redes existentes se aplica para mejorar características operativas como perfiles de tensión o reducción de pérdidas por efecto joule, o para sustituir de manera eficiente redes que han cumplido su vida útil [4].
Dada la importancia que tiene el sistema de distribución para la correcta atención de la demanda, es necesario que las DisCo cuenten con herramientas eficientes para apoyar a los expertos de planeación en la toma de decisiones sobre la ampliación del sistema a un mínimo costo, de forma que sea eficiente y confiable [4]. De acuerdo con esto, la selección óptima de conductores ha sido un problema de amplia acogida en la literatura especializada. Dentro de los trabajos más destacados en este campo se resalta la aplicación de algoritmos heurísticos [6-7], técnicas de optimización combinatorial como algoritmos genéticos [4] y estrategias evolutivas [8-10]. Otros autores han propuesto modelos lineales [11] y no lineales [12] para representar el problema, los que son resueltos por medio de técnicas exactas disponibles en software de optimización comercial.

A diferencia de los trabajos anteriores, en este artículo se propone una metodología de solución al problema de selección óptima de conductores en sistemas de distribución (SD) empleando la técnica de optimización búsqueda tabú (BT) que considera la curva de duración de carga anual del sistema de distribución discretizada en tres períodos, ya que en contraste con los algoritmos genéticos, esta técnica es menos susceptible a caer en espacios infactibles, debido a que el recorrido por el espacio de solución se hace de manera controlada mediante los criterios de vecindad y no por medio de mutaciones o combinaciones aleatorias de los individuos, que en general producen altos índices de infactibilidad en este tipo de problemas.

Los resultados presentados en este trabajo son de mejor calidad que los reportados en la literatura especializada [4], muestran la eficiencia, robustez y aplicabilidad de la metodología propuesta en SD con una o múltiples fuentes, garantizando siempre la radialidad de la red. Adicionalmente, el modelo matemático desarrollado es fácilmente adaptable a los períodos de análisis que la DisCo considere adecuados en sus planes de expansión y/o reposición de redes.

Finalmente, este documento está organizado como sigue. En la sección 2 se presenta la formulación matemática del problema. En la sección 3 se describe la técnica de optimización BT y sus principales características. En la sección 4 se muestra la aplicación de la metodología propuesta a dos 
sistemas de prueba de la literatura y los resultados obtenidos. La sección 5 presenta las conclusiones derivadas de este trabajo y, por último, se encuentran las referencias bibliográficas.

\section{FORMULACIÓN MATEMÁTICA}

Para describir matemáticamente el problema de selección óptima de conductores en sistemas de distribución, es empleado un modelo de programación no lineal entero mixto no convexo monoobjetivo como el presentado en [3].

El modelo matemático propuesto se describe de (1) a (8).

$\min z=3\left(\begin{array}{c}C_{W h} \cdot \sum_{i j \in \Omega_{L}} \sum_{c \in \Omega C} \sum_{h \in \Omega_{H}} L_{i j} \cdot \delta_{i j}^{c} \cdot D C C_{h} \cdot r_{i j}^{c} \cdot\left(I_{i j, h}^{c}\right)^{2} \\ +\sum_{i j \in \Omega_{L}} \sum_{c \in \Omega C} L_{i j} \cdot \delta_{i j}^{c} \cdot C I C^{c}\end{array}\right)$

s.a.

$P_{i, h}^{G}-P_{i, h}^{D}=\sum_{j \in \Omega_{L}} \sum_{c \in \Omega_{C}} \delta_{i j}^{c} \cdot P_{i j, h}^{c}\left\{\forall i \in \Omega_{N}, \forall h \in \Omega_{H}\right\}_{(2)}$

$Q_{i, h}^{G}-Q_{i, h}^{D}=\sum_{j \in \Omega_{L}} \sum_{c \in \Omega_{C}} \delta_{i j}^{c} \cdot Q_{i j, h}^{c}\left\{\forall i \in \Omega_{N}, \forall h \in \Omega_{H}\right\}$

$V_{i}^{\min } \leq V_{i, h} \leq V_{i}^{\max }\left\{\forall i \in \Omega_{N}, \forall h \in \Omega_{H}\right\}$

$\sum_{c \in \Omega_{c}} \delta_{i j}^{c} \cdot\left(\left(I_{i j, h}^{r, c}\right)^{2}+\left(I_{i j, h}^{i, c}\right)^{2}\right) \leq \sum_{c \in \Omega_{c}} \delta_{i j}^{c}$

$\left(I_{i j}^{c, \max }\right)^{2}\left\{\forall i j \in \Omega_{L}, \forall h \in \Omega_{H}\right\}$

$$
\begin{gathered}
\sum_{k l \in \Omega_{L}} \sum_{c \in \Omega_{c}} \delta_{k l}^{c} \cdot a^{c} \cdot T_{i j, k l} \geq\left(\sum_{c \in \Omega_{c}} \delta_{i j}^{c} \cdot a^{c}\right) . \\
\left(\sum_{k l \in \Omega_{L}} T_{i j, k l}\right)\left\{\forall i j \in \Omega_{L}\right\}
\end{gathered}
$$$$
\sum_{c \in \Omega_{c}} \delta_{i j}^{c}=1\left\{\forall i j \in \Omega_{L}\right\}
$$

$$
\delta_{i j}^{c} \in\{0,1\}\left\{\forall i j \in \Omega_{L}\right\}
$$

La función objetivo presentada en (1) corresponde a una función de costos de inversión y operación evaluados para el período de estudio (un año), en donde la primera componente representa las pérdidas técnicas producidas por el calentamiento de los conductores debido a la circulación de corriente y la segunda componente el costo de inversión asociado a los calibres de los conductores. Por otro lado, para la correcta operación del sistema de distribución, son consideradas las restricciones de balance de potencia activa y reactiva así como se muestra en (2) y (3), la regulación de tensión en los nodos y la capacidad térmica de los tramos de red se determina según (4) y (5), respectivamente.

Para garantizar que la red resultante sea de naturaleza telescópica se propone la expresión (6) la que determina el conjunto necesario de ecuaciones asociadas al número de tramos de red $k l$ que se encuentra inmediatamente aguas arriba del tramo de red $i j$.

La restricción (7) implica que en cada tramo de red $i j$ debe existir un conductor con calibre tipo c. Por último, (8) define la naturaleza binaria de las variables de decisión.

En el conjunto de ecuaciones (9) - (13) se presenta la forma de calcular los flujos de potencia activa y reactiva, además de las componentes real e imaginaria de la corriente, respectivamente, como una función de la variable de decisión y las variables de estado del sistema de distribución, es decir, las magnitudes y los ángulos de los voltajes en cada nodo.

$$
\begin{gathered}
P_{i j, h}^{c}=V_{i, h}^{2} \cdot g_{i j}^{c}-V_{i, h} \cdot V_{j, h} \cdot\left(\begin{array}{l}
g_{i j}^{c} \cdot \cos \left(\theta_{i j, h}\right)+ \\
b_{i j}^{c} \cdot \sin \left(\theta_{i j, h}\right)
\end{array}\right) \\
Q_{i j, h}^{c}=-V_{i, h}^{2} \cdot b_{i j}^{c}-V_{i, h} \cdot\left(\begin{array}{l}
g_{i j}^{c} \cdot \sin \left(\theta_{i j, h}\right)- \\
b_{i j}^{c} \cdot \cos \left(\theta_{i j, h}\right)
\end{array}\right) \\
I_{i j, h}^{r, c}=\left\{\begin{array}{l}
g_{i j}^{c} \cdot\left(V_{i, h} \cos \left(\theta_{i, h}\right)-V_{j, h} \cdot \cos \left(\theta_{j, h}\right)\right)- \\
b_{i j}^{c} \cdot\left(V_{i, h} \cdot \sin \left(\theta_{i, h}\right)-V_{j, h} \cdot \sin \left(\theta_{j, h}\right)\right)
\end{array}\right\} \\
I_{i j, h}^{i, c}=\left\{\begin{array}{l}
g_{i j}^{c} \cdot\left(V_{i, h} \cdot \sin \left(\theta_{j, h}\right) V_{j, h} \cdot \sin \left(\theta_{j, h}\right)\right)+ \\
b_{i j}^{c} \cdot\left(V_{i, h} \cdot \cos \left(\theta_{i, h}\right) V_{j, h} \cdot \cos \left(\theta_{j, h}\right)\right)
\end{array}\right\}
\end{gathered}
$$




$$
\begin{aligned}
g_{i j}^{c}= & \frac{1}{L_{i j}} \cdot \frac{r_{i j}^{c}}{\left(r_{i j}^{c}\right)^{2}+\left(x_{i j}^{c}\right)^{2}} \wedge b_{i j}^{c}= \\
& -\frac{1}{L_{i j}} \cdot \frac{x_{i j}^{c}}{\left(r_{i j}^{c}\right)^{2}+\left(x_{i j}^{c}\right)^{2}}
\end{aligned}
$$

\section{ALGORITMO BÚSQUEDA TABÚ}

\section{Descripción general del BT}

El algoritmo BT es una técnica de optimización matemática perteneciente a la familia de las técnicas metaheurísticas de optimización, que en términos generales fue diseñada para encontrar configuraciones óptimas (solución global) o soluciones de buena calidad (solución local), en problemas de alta complejidad matemática (problemas no convexos, no diferenciables y con variables mixtas), y de característica combinatorial, en los que su espacio de solución crece exponencialmente con el tamaño del problema [13-14]. En general, el algoritmo BT resuelve problemas de optimización monoobjetivo de la forma (14). Es de notarse que la función objetivo puede tratarse de una función a maximizar o minimizar, según sea la naturaleza del problema [1-2].

$$
\begin{aligned}
& z=\min (f(x)) \\
& \text { s.a. } \\
& h(x)=0 \wedge g(x) \leq 0\{\forall x \in S\}
\end{aligned}
$$

El algoritmo BT realiza una búsqueda por el espacio de solución por medio de un recorrido de trayectoria, como lo hace un algoritmo heurístico de búsqueda local [2]. Así, dada la configuración actual $x^{t}$ se define el vecindario $N\left(x^{t}\right)$ como el conjunto de elementos adyacentes a $x^{t}$ tal que $x^{t} \in N\left(x^{t}\right)$; cuando el vecindario es muy grande, se emplean criterios basados en el conocimiento del problema para obtener de $N\left(x^{t}\right)$ un vecindario reducido $N^{\prime}\left(x^{t}\right)$. Este conjunto de vecinos puede ser obtenido por medio de estrategias heurísticas guiadas o mediante un proceso de generación aleatorio. Sin embargo, el BT se diferencia de un algoritmo heurístico de búsqueda local en tres aspectos esenciales [13-14]:

- En la selección de la configuración siguiente $x^{t+1}$ se selecciona el mejor vecino encontrado, si este tiene mejor función de adaptación que la configuración asociada $x^{t}$, aplicando lo que se denomina criterio de aspiración; en caso contrario, se selecciona aquel individuo del vecindario que tenga menos atributos bloqueados por los criterios tabú, lo que permite degradaciones en la función de adaptación, con el fin de escapar de soluciones locales y explorar otras regiones.

- Son empleadas memorias de corto y largo plazo que impiden que la búsqueda regrese a espacios de solución anteriormente explorados en las búsquedas locales.

- Es empleada una estrategia de reinicio para comenzar de nuevo la búsqueda local, con la que se busca explorar el espacio de solución desde puntos de arranque diversos, que garanticen alcanzar una solución de buena calidad.

La solución óptima (mejor solución encontrada) se encuentra contenida en la lista élite, la cual almacena las mejores soluciones obtenidas en las búsquedas locales efectuadas según el proceso antes mencionado. El pseudocódigo presentado en la Figura 1 muestra el proceso básico del BT mientras recorre el espacio de solución.

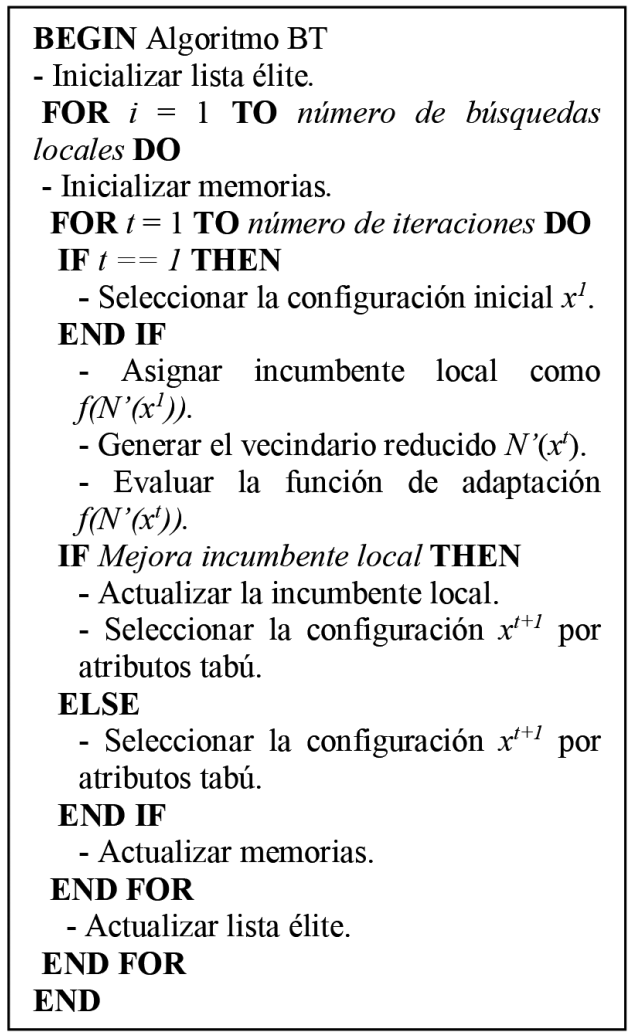

Figura 1. Pseudocódigo del algoritmo BT [1]. 
En el algoritmo BT existen otras estrategias de búsqueda avanzada, entre las que se destacan la oscilación estratégica y el encadenamiento de trayectorias, cuyos detalles y la forma de implementación pueden ser consultadas en [13-14].

\section{Configuración inicial}

Como el problema de asignación óptima de conductores en SD dada su naturaleza, conserva una configuración topológica invariante en el tiempo (las rutas de las líneas no son una variable de decisión, sino un parámetro de entrada), la configuración inicial para los calibres se determina de dos formas diferentes:

- Es evaluado un flujo de potencia del tipo barrido iterativo [15] asumiendo como valores de resistencia y reactancia para cada tramo de red $i j$, igual al promedio de los valores de entrada asociados a los calibres de conductores disponibles; una vez conocidas las corrientes promedio en cada línea, se procede a asignar los calibres por cada alimentador, desde el nodo fuente hacia los nodos finales, tomando como referente el calibre con capacidad térmica inmediatamente superior al valor de corriente registrada en la línea.

- Es asignado de manera aleatoria un calibre para toda la red, con base en los datos disponibles en los conductores de entrada y posteriormente evaluado el flujo de potencia para verificar capacidades en conductores; si la capacidad no es excedida en ningún tramo de red, esta se toma como la configuración inicial, en caso contrario, se asigna el siguiente calibre (mayor capacidad de conducción) para todos los tramos de red y nuevamente se verifica la capacidad térmica. La configuración inicial será aquella que con calibre uniforme no viole los límites de corriente en los conductores.

\section{Generación del vecindario reducido}

Para la generación del vecindario reducido $N^{\prime}\left(x^{t}\right)$ asociado a la configuración actual $x^{t}$, son empleados cuatro estrategias heurísticas basadas en las características operativas de la red [1-2], las que se describen a continuación:

- Es creada una lista con todos los recorridos que existen entre los nodos finales y la subestación. Para cada recorrido es seleccionado de manera aleatoria un tramo de red y es variado su calibre; posteriormente son cambiados los demás calibres de dicho alimentador, hasta garantizar una configuración telescópica.

- Son determinados los nodos con peor regulación. Para los recorridos entre cada nodo y la subestación es incrementado el calibre al siguiente de mayor capacidad.

- Es seleccionado el tramo de red con menor cargabilidad porcentual. Para cada tramo es disminuido el calibre al siguiente de menor capacidad; aguas abajo de este son modificados todos aquellos que violen la característica de red telescópica.

- Es escogido de forma aleatoria un alimentador y luego a sus tramos de red se les asigna el mismo tipo de conductor.

Si el total de vecinos supera un número máximo permitido $k$, este es reducido por medio de la selección aleatoria de los primeros $k$ vecinos; si el vecindario generado es menor que el número $k$, para esa generación se trabaja con dicha cantidad. Lo anterior indica que el BT implementado tiene un tamaño dinámico en el vecindario, variando este entre 2 y $k$ vecinos.

\section{Codificación empleada}

Para representar adecuadamente el problema de selección óptima de conductores en $\mathrm{SD}$, es empleado un vector binario con dimensiones $N_{L} * N_{C}$, donde $N_{L}$ es el número de tramos de red y $N_{C}$ es el número total de calibres disponibles. La Figura 2 muestra la codificación empleada.

\begin{tabular}{|c|c|c|c|c|c|c|c|c|}
\hline \multicolumn{2}{|c|}{$\begin{array}{c}\text { Tramo de } \\
\text { red } I \\
\end{array}$} & \multicolumn{3}{|c|}{$\begin{array}{c}\text { Tramo de } \\
\text { red } 2 \\
\end{array}$} & & \multicolumn{3}{|c|}{$\begin{array}{c}\text { Tramo de } \\
\operatorname{red} N_{i j} \\
\ell\end{array}$} \\
\hline$\delta_{1}^{1}$ & $\delta_{1}^{c}$ & $\delta_{2}^{1}$ & & $\delta_{2}^{c}$ & & $\delta_{N i j}^{1}$ & & $\delta_{N i j}^{c}$ \\
\hline 1 & 0 & 1 & l & 0 & & 1 & 0 & 0 \\
\hline
\end{tabular}

Figura 2. Codificación empleada [1].

\section{Criterios de parada}

En al algoritmo BT son empleados dos estrategias de exploración sobre el espacio de solución conocidas como búsqueda global y búsqueda local. Las búsquedas globales corresponden a la cantidad de puntos diferentes sobre el espacio de solución que son generados como punto de partida para las búsquedas locales. Por otro lado, las exploraciones 
locales corresponden a múltiples búsquedas realizadas desde el mismo punto de inicialización.

En este sentido, son empleados dos criterios de parada en cada búsqueda local. El primero corresponde al límite de generaciones de cada búsqueda definido en la parametrización del BT y el segundo criterio emplea el número de veces en que el algoritmo no mejora la incumbente. La mejor solución encontrada en la búsqueda local es almacenada en la lista élite global [14].

Para el caso de las búsquedas globales son realizadas como mínimo dos sobre el espacio de solución, en caso de la segunda búsqueda obtenga una mejor función objetivo que la primera, se inicia una nueva búsqueda global y este proceso continua hasta que la incumbente global desmejore en alguna de las búsquedas globales.

\section{Función de adaptación}

Dado que una de las estrategias del BT es explorar espacios de solución con algún grado de infactibilidad, la función de adaptación empleada para la evolución del BT es manejada por medio de penalizaciones [1-2]. La ecuación (15) muestra la función de adaptación implementada, donde $z$ es la función objetivo definida en (1).

$$
\tilde{z}=\min \left(\begin{array}{l}
z+\alpha_{1} \cdot \operatorname{máx}\left\{0, I_{i j, h}^{c}-I_{i j}^{c, \text { máx }}\right\} \\
+\alpha_{2} \cdot \operatorname{máx}\left\{0, V_{i}^{\min }-V_{i, h}\right\}
\end{array}\right)
$$

$\alpha_{1}$ y $\alpha_{2}$ corresponden a los factores de penalización asociados a la máxima desviación por corrientes y por tensiones, respectivamente. La penalización que se propone es del tipo diferencial, es decir, en cuanto menor es la diferencia en corrientes y/o voltajes, menor es la penalización. La función $\max \left\{0, I_{i j, h}^{c}-I_{i j}^{c, \text { máx }}\right\}$ determina el tramo $i j$ con mayor diferencia entre la corriente obtenida por medio de la evaluación del flujo de potencia y su límite térmico, para cada período $h$; de igual forma se realiza para los voltajes en todos los nodos del sistema.

\section{APLICACIÓN Y RESULTADOS}

Para implementar la metodología propuesta, es empleado el software MATLAB 2015a, en un computador con procesador intel core $i 3,4 \mathrm{~Gb}$ de RAM y Windows 7 Professional.

\section{Escenarios de simulación y parámetros considerados}

Para evaluar la metodología propuesta son considerados dos escenarios para la potencia demandada. El primer escenario $\left(\mathrm{E}_{1}\right)$ corresponde a la operación en demanda pico para todo el año de estudio. El segundo escenario $\left(E_{2}\right)$, corresponde a la evaluación de las pérdidas bajo una curva de carga discretizada en tres períodos de demanda, así como se muestra en la Tabla 1.

Tabla 1. Curva de carga de discretizada.

\begin{tabular}{|c|r|r|r|}
\hline Parámetro & Alta & Media & \multicolumn{1}{c|}{ Baja } \\
\hline Demanda [\%] & 100 & 60 & 30 \\
\hline Duración [h] & 1.000 & 6.760 & 1.000 \\
\hline
\end{tabular}

Los escenarios se proponen con el fin de realizar diversos análisis comparativos entorno a respuestas disponibles en la literatura especializada y como posible herramienta para implementación dentro de un problema de planeación de mayor envergadura.

Los tipos de conductores, costos y características técnicas para efectos comparativos, fueron tomadas de [4] y se muestran en la Tabla 2.

Es importante resaltar que la información asociada a los costos de los conductores ha sido convertida de reales brasileños a dólares americanos con una tasa de cambio correspondiente a US\$ $1=\mathrm{R} \$ 3.22$.

Para la implementación del algoritmo BT y la metodología propuesta son empleados los parámetros que se presentan en la Tabla 3.

Es de tener en cuenta que los parámetros presentados en la Tabla 3 han sido ajustados mediante un algoritmo heurístico de ensayo y error después de realizar múltiples simulaciones con diferentes valores de entrada.

\section{Sistemas de prueba}

Para validar la metodología propuesta son empleados dos sistemas de prueba disponibles en la literatura especializada. El primero corresponde a un sistema de $13,8 \mathrm{kV}$, con una subestación, 3 alimentadores, 8 nodos de demanda con factor de potencia unitario y 7 tramos de red propuestos. La Tabla 4 muestra la información de los tramos de red y su topología se observa en la Figura 3. 
Tabla 2. Características de los conductores.

\begin{tabular}{|c|c|c|c|c|c|}
\hline Tipo $\boldsymbol{\alpha}^{\mathbf{c}}$ & Calibre & $\mathbf{R}[\mathbf{\Omega} / \mathbf{k m}]$ & $\mathbf{X}[\mathbf{\Omega} / \mathbf{k m}]$ & $\mathbf{I m a x}[\mathbf{A}]$ & Costos [USD\$] \\
\hline 1 & 2 & 0,8763 & 0,4133 & 180 & 1.986 \\
\hline 2 & 1 & 0,6960 & 0,4133 & 200 & 2.790 \\
\hline 3 & $1 / 0$ & 0,5518 & 0,4077 & 230 & 3.815 \\
\hline 4 & $2 / 0$ & 0,4387 & 0,3983 & 270 & 5.090 \\
\hline 5 & $3 / 0$ & 0,3480 & 0,3899 & 300 & 8.067 \\
\hline 6 & $4 / 0$ & 0,2765 & 0,3610 & 340 & 12.673 \\
\hline 7 & 336 & 0,0966 & 0,1201 & 600 & 23.419 \\
\hline 8 & 556 & 0,0853 & 0,0950 & 720 & 30.070 \\
\hline
\end{tabular}

Tabla 3. Parámetros empleados en el BT.

\begin{tabular}{|c|c|l|c|}
\hline Parámetro & Valor & \multicolumn{1}{c|}{ Parámetro } & Valor \\
\hline$\alpha_{1}$ & 2,000 & Iteraciones & 30 \\
\hline$\alpha_{2}$ & 1,000 & Parador local & 1,000 \\
\hline$B_{L}$ & 100 & Regulación de tensión [\%] & \pm 5 \\
\hline Costo energía [US\$/kWh] & 0,078 & Tamaño del vecindario & $2-10$ \\
\hline
\end{tabular}

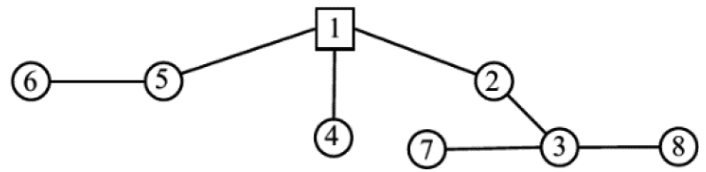

Figura 3. Sistema 8 nodos [4].

Tabla 4. Información de tramos de red y demanda para el sistema de prueba 1 .

\begin{tabular}{|c|c|c|c|c|c|c|}
\hline $\boldsymbol{N}_{\boldsymbol{i}}$ & $\boldsymbol{N}_{\boldsymbol{j}}$ & $\begin{array}{c}\boldsymbol{L}_{\boldsymbol{i j}} \\
{[\mathbf{k m}]}\end{array}$ & $\boldsymbol{P D _ { j } [ \mathbf { k W ] } ]}$ & $\begin{array}{c}\boldsymbol{Q D D _ { j }} \\
{[\mathbf{k V A r}]}\end{array}$ & $\begin{array}{c}\boldsymbol{E}_{\boldsymbol{1}} \\
\text { Calibre }\end{array}$ & $\begin{array}{c}\boldsymbol{E}_{\boldsymbol{2}} \\
\text { Calibre }\end{array}$ \\
\hline 1 & 2 & 1 & $1.054,2$ & 0 & 6 & 6 \\
\hline 2 & 3 & 1 & 806,50 & 0 & 5 & 4 \\
\hline 1 & 4 & 1 & $2.632,5$ & 0 & 4 & 3 \\
\hline 1 & 5 & 1 & 609 & 0 & 4 & 3 \\
\hline 5 & 6 & 1 & $2.034,5$ & 0 & 4 & 2 \\
\hline 3 & 7 & 1 & 932,80 & 0 & 1 & 1 \\
\hline 3 & 8 & 1 & $1.731,4$ & 0 & 3 & 2 \\
\hline
\end{tabular}

El segundo sistema de prueba corresponde a una adaptación del sistema de $13.2 \mathrm{kV}$ con 202 nodos. Este sistema cuenta con 2 subestaciones y 5 alimentadores. La topología de este sistema puede ser consultada en [17] y los datos de demanda y tramos de red se presentan en las Tablas 5 y 6 .

\section{Resultados $E_{1}$}

Para el sistema de prueba 1 se encontraron 9 soluciones con menor valor respecto a los reportados en [4], en la Tabla 7 se muestran los resultados comparativos, respecto a la referencia.

Al observar los datos de la Tabla 7 es claro que todas las soluciones tienen un costo total inferior al presentado en [4]; sin embargo, al detallar la segunda columna (costo de las pérdidas), se nota que todas las soluciones tienen un mayor valor en esta componente de la función objetivo. No obstante, la tercera columna (costo de los conductores) muestra un comportamiento opuesto, situación que es natural, ya que un menor calibre de conductor presenta mayores pérdidas.

La mejor solución encontrada (respuesta 1 de la Tabla 7), presenta, en relación con [4], una reducción en el costo operativo equivalente a $1,11 \%$ y su configuración en conductores se observa en la columna $\boldsymbol{E}_{\mathbf{1}}$ de la Tabla 4, mientras que la respuesta 7 de la Tabla 7 presenta una reducción de tan solo el $0,03 \%$.

Por otro lado, para todas las respuestas presentadas en la Tabla 4 la peor regulación de tensión es menor al $2 \%$, lo que garantiza calidad y eficiencia en los perfiles de tensión con los que se atiende la demanda.

En el caso del sistema de prueba 2, la mejor solución encontrada tiene como función objetivo US\$ 924.379,65 distribuidos US\$ 413.169,44 asociados a pérdidas técnicas y US\$ 511.210,21 
Tabla 5. Información de los tramos de red para el sistema de prueba 2.

\begin{tabular}{|c|c|c|c|c|c|c|c|c|c|c|c|}
\hline $\mathbf{N}_{\mathbf{i}}$ & $\mathbf{N}_{\mathrm{j}}$ & $L_{i j} Z[\mathbf{k m}]$ & $\mathbf{N}_{\mathbf{i}}$ & $\mathbf{N}_{\mathrm{j}}$ & $L_{i j}[\mathrm{~km}]$ & $\mathbf{N}_{\mathbf{i}}$ & $\mathbf{N}_{\mathrm{j}}$ & $L_{i j} \mathbf{Z}[\mathbf{k m}]$ & $\mathbf{N}_{\mathbf{i}}$ & $\mathbf{N}_{\mathrm{j}}$ & $L_{i j}[\mathrm{~km}]$ \\
\hline 201 & 91 & 1.064 & 19 & 50 & 240 & 199 & 200 & 20 & 56 & 146 & 430 \\
\hline 201 & 189 & 600 & 102 & 109 & 210 & 115 & 96 & 120 & 56 & 124 & 250 \\
\hline 201 & 122 & 70 & 100 & 99 & 160 & 113 & 114 & 130 & 148 & 145 & 150 \\
\hline 91 & 1 & 933 & 105 & 110 & 180 & 14 & 10 & 455 & 146 & 139 & 420 \\
\hline 91 & 86 & 44 & 6 & 5 & 110 & 174 & 180 & 290 & 145 & 144 & 90 \\
\hline 189 & 158 & 1.000 & 8 & 7 & 260 & 169 & 173 & 100 & 202 & 69 & 850 \\
\hline 189 & 188 & 200 & 140 & 129 & 160 & 164 & 172 & 380 & 69 & 65 & 175 \\
\hline 122 & 119 & 1.250 & 140 & 152 & 250 & 117 & 83 & 110 & 69 & 58 & 147 \\
\hline 1 & 12 & 110 & $\begin{array}{l}153 \\
153\end{array}$ & 133 & 270 & 157 & 123 & 280 & 65 & 67 & 180 \\
\hline $\begin{array}{r}1 \\
158\end{array}$ & $\begin{array}{r}13 \\
183\end{array}$ & $\begin{array}{r}45 \\
620\end{array}$ & $\begin{array}{l}153 \\
150\end{array}$ & $\begin{array}{l}155 \\
151\end{array}$ & $\frac{110}{150}$ & 96 & 116 & 110 & 58 & 76 & 1060 \\
\hline 158 & 181 & 280 & 150 & 154 & 190 & 114 & 111 & 110 & 58 & 68 & 58 \\
\hline 158 & 160 & 230 & 22 & 27 & 190 & 10 & 9 & 160 & 67 & 66 & 236 \\
\hline 158 & 198 & 740 & 52 & 25 & 275 & 10 & 85 & 120 & 67 & 84 & 80 \\
\hline 188 & 197 & 300 & 43 & 31 & 700 & 180 & 159 & 250 & 67 & 92 & 230 \\
\hline 188 & 192 & 64 & 43 & 24 & 350 & 180 & 177 & 160 & 76 & 72 & 210 \\
\hline 119 & 93 & 1.076 & 50 & 51 & 190 & 83 & 103 & 200 & 76 & 77 & 140 \\
\hline 12 & 3 & 155 & 50 & 53 & 150 & 83 & 108 & 130 & 66 & 63 & 123 \\
\hline 13 & 15 & 270 & 99 & 101 & 150 & 83 & 98 & 360 & 72 & 71 & 270 \\
\hline 183 & 186 & 120 & 5 & 4 & 135 & 83 & 32 & 115 & 72 & 79 & 200 \\
\hline 183 & 162 & 260 & 7 & 18 & 60 & 83 & 106 & 180 & 77 & 78 & 60 \\
\hline 181 & 182 & 90 & 129 & 130 & 150 & 123 & 126 & 80 & 63 & 61 & 165 \\
\hline 181 & 185 & 250 & 152 & 131 & 240 & 116 & 95 & 215 & 71 & 73 & 60 \\
\hline 160 & 184 & 360 & 133 & 138 & 410 & 111 & 94 & 90 & 71 & 20 & 530 \\
\hline 160 & 171 & 240 & 155 & 136 & 230 & 9 & 87 & 375 & 79 & 81 & 200 \\
\hline 198 & 196 & 190 & 151 & 134 & 210 & 9 & 2 & 295 & 78 & 82 & 660 \\
\hline 198 & 191 & 40 & 154 & 137 & 290 & 159 & 128 & 780 & 61 & 57 & 354 \\
\hline 197 & 195 & 100 & 27 & 35 & $\begin{array}{l}250 \\
150\end{array}$ & 159 & 179 & 120 & 73 & 74 & 290 \\
\hline $\begin{array}{l}93 \\
93\end{array}$ & $\frac{118}{97}$ & 290 & 25 & $\frac{29}{36}$ & $\frac{150}{290}$ & 103 & 104 & 170 & 20 & 46 & 230 \\
\hline $\begin{array}{l}93 \\
93\end{array}$ & $\begin{array}{r}91 \\
112\end{array}$ & $\frac{110}{90}$ & $\frac{31}{24}$ & $\frac{36}{34}$ & $\frac{290}{350}$ & 108 & 107 & 170 & 20 & 48 & 370 \\
\hline 3 & 11 & 230 & 51 & 55 & 320 & 32 & 54 & 120 & 20 & 49 & 100 \\
\hline 186 & 166 & 290 & 4 & 17 & 335 & 32 & 21 & 120 & 82 & 89 & 331 \\
\hline 182 & 161 & 230 & 130 & 156 & 100 & 32 & 19 & 180 & 57 & 59 & 30 \\
\hline 185 & 165 & 160 & 131 & 135 & 410 & 106 & 102 & 150 & 74 & 80 & 230 \\
\hline 184 & 163 & 250 & 138 & 142 & 170 & 126 & 127 & 740 & 46 & 33 & 475 \\
\hline 171 & 187 & 250 & 136 & 143 & 260 & 95 & 100 & 290 & 46 & 39 & 250 \\
\hline 196 & 190 & 30 & 134 & 194 & 25 & 94 & 105 & 230 & 48 & 44 & 120 \\
\hline 118 & 121 & 140 & 137 & 193 & 35 & 87 & 88 & 220 & 49 & 47 & 200 \\
\hline 118 & 199 & 210 & 29 & 38 & 320 & 2 & 6 & 142 & 49 & 45 & 100 \\
\hline 118 & 120 & 35 & 36 & 41 & 185 & 2 & 8 & 40 & 59 & 60 & 120 \\
\hline 97 & 115 & 170 & 55 & 26 & 260 & 128 & 140 & 110 & 33 & 28 & 315 \\
\hline 112 & 113 & 210 & 156 & 132 & 310 & 128 & 153 & 120 & 47 & 42 & 220 \\
\hline $\begin{array}{r}11 \\
166\end{array}$ & $\begin{array}{r}14 \\
174\end{array}$ & $\begin{array}{l}360 \\
310\end{array}$ & $\begin{array}{l}135 \\
143\end{array}$ & $\begin{array}{l}141 \\
147\end{array}$ & $\begin{array}{l}220 \\
260\end{array}$ & 128 & 150 & 90 & 60 & 62 & 300 \\
\hline 165 & 169 & 160 & 26 & 30 & 280 & 179 & 176 & 150 & 28 & 23 & 260 \\
\hline 163 & 164 & 90 & 147 & 56 & 400 & 54 & 22 & 210 & 42 & 40 & 320 \\
\hline 121 & 117 & 550 & 147 & 149 & 60 & 21 & 52 & 110 & 62 & 70 & 62 \\
\hline 199 & 157 & 60 & 56 & 148 & 190 & 19 & 43 & 40 & 70 & 64 & 195 \\
\hline
\end{tabular}


Tabla 6. Información de demanda para el sistema de prueba 2.

\begin{tabular}{|c|c|c|c|c|c|c|c|c|c|c|c|}
\hline Nodo $i$ & $\begin{array}{c}P D_{i} \\
{[k W]}\end{array}$ & $\underset{[\mathbf{k V A}]}{Q D_{i}}$ & Nodo $i$ & $\begin{array}{c}P \boldsymbol{D}_{\boldsymbol{i}} \\
{[\mathrm{kW}]}\end{array}$ & $\underset{[\mathbf{k V A}]}{Q D_{i}}$ & Nodo $i$ & $\begin{array}{c}P D_{i} \\
{[k W]}\end{array}$ & $\underset{[\mathbf{k V A}]}{Q D_{i}}$ & Nodo $i$ & $\begin{array}{c}P D_{i} \\
{[\mathbf{k W}]}\end{array}$ & $\underset{[\mathbf{k V A}]}{Q Q D_{i}}$ \\
\hline 1 & 0 & 0 & 102 & 37 & 19 & 50 & 81 & 41 & 151 & 81 & 41 \\
\hline 2 & 81 & 41 & 103 & 25 & 13 & 51 & 200 & 120 & 152 & 0 & 0 \\
\hline 3 & 80 & 60 & 104 & 14 & 7 & 52 & 28 & 14 & 153 & 150 & 80 \\
\hline 4 & 50 & 30 & 105 & 80 & 100 & 53 & 81 & 41 & 154 & 50 & 30 \\
\hline 5 & 76 & 38 & 106 & 52 & 26 & 54 & 8 & 4 & 155 & 35 & 17 \\
\hline 6 & 34 & 17 & 107 & 81 & 41 & 55 & 52 & 26 & 156 & 130 & 65 \\
\hline 7 & 55 & 28 & 108 & 25 & 13 & 56 & 33 & 17 & 157 & 7 & 4 \\
\hline 8 & 0 & 0 & 109 & 81 & 41 & 57 & 0 & 0 & 158 & 85 & 43 \\
\hline 9 & 81 & 41 & 110 & 18 & 9 & 58 & 40 & 20 & 159 & 11 & 5 \\
\hline 10 & 14 & 7 & 111 & 43 & 22 & 59 & 47 & 24 & 160 & 25 & 13 \\
\hline 11 & 52 & 26 & 112 & 0 & 0 & 60 & 81 & 41 & 161 & 103 & 52 \\
\hline 12 & 0 & 0 & 113 & 0 & 0 & 61 & 52 & 26 & 162 & 52 & 26 \\
\hline 13 & 45 & 23 & 114 & 0 & 0 & 62 & 64 & 32 & 163 & 18 & 9 \\
\hline 14 & 47 & 24 & 115 & 0 & 0 & 63 & 80 & 40 & 164 & 68 & 34 \\
\hline 15 & 90 & 40 & 116 & 0 & 0 & 64 & 52 & 26 & 165 & 81 & 41 \\
\hline 16 & 52 & 26 & 117 & 53 & 26 & 65 & 68 & 34 & 166 & 48 & 24 \\
\hline 17 & 20 & 10 & & 164 & 82 & 66 & 35 & 17 & 167 & 81 & 41 \\
\hline 18 & 8 & 4 & & 0 & 0 & 67 & 38 & 19 & 168 & 130 & 65 \\
\hline 19 & 12 & 10 & & 40 & 30 & 68 & 114 & 57 & 169 & 52 & 26 \\
\hline 20 & 130 & 65 & $\begin{array}{l}120 \\
121\end{array}$ & 80 & 30 & 69 & 80 & 40 & 170 & 55 & 28 \\
\hline 21 & 52 & & 121 & 90 & 50 & 70 & 26 & 13 & 171 & 35 & 18 \\
\hline 22 & 52 & & 122 & 150 & 40 & $\frac{71}{72}$ & $\begin{array}{l}79 \\
32\end{array}$ & $\frac{40}{16}$ & $\frac{172}{173}$ & $\frac{81}{43}$ & $\frac{41}{22}$ \\
\hline 23 & 52 & 26 & $\frac{123}{124}$ & 52 & 26 & 73 & 33 & 17 & 174 & $\begin{array}{l}45 \\
58\end{array}$ & 22 \\
\hline 24 & 81 & 41 & 125 & $\frac{01}{41}$ & $\frac{4}{2}$ & 74 & 29 & 15 & 175 & 55 & 28 \\
\hline 25 & 70 & 35 & 126 & $\begin{array}{l}41 \\
70\end{array}$ & $\frac{4}{3}$ & 75 & 55 & 28 & 176 & 0 & 0 \\
\hline 26 & 90 & 10 & 127 & 81 & $\frac{3}{41}$ & 76 & 88 & 44 & 177 & 112 & 56 \\
\hline 27 & 52 & 26 & 128 & $\begin{array}{l}01 \\
52\end{array}$ & $\frac{41}{26}$ & 77 & 130 & 65 & 178 & 0 & 0 \\
\hline 28 & 162 & 81 & 129 & 52 & $\frac{20}{26}$ & 78 & 60 & 20 & 179 & 0 & 0 \\
\hline 29 & 52 & 26 & 130 & 0 & $\frac{20}{0}$ & 79 & 81 & 41 & 180 & 88 & 44 \\
\hline 30 & 52 & 26 & 131 & 56 & 28 & 80 & 2 & 1 & 181 & 14 & 7 \\
\hline 31 & 0 & 0 & 132 & 5 & & 81 & 35 & 17 & 182 & 14 & 7 \\
\hline 32 & 80 & 40 & 133 & 81 & 41 & 82 & 68 & 34 & 183 & 14 & 7 \\
\hline 33 & 130 & 65 & 134 & 23 & & 83 & 14 & 7 & 184 & 14 & 7 \\
\hline 34 & 81 & 41 & 135 & 37 & & 84 & 14 & 1 & 185 & 40 & 20 \\
\hline 35 & 52 & 26 & 136 & 102 & & 85 & 14 & 7 & 186 & 30 & 10 \\
\hline 36 & 100 & 60 & 137 & 211 & $\begin{array}{r}J 1 \\
106\end{array}$ & 86 & 4 & $\frac{2}{0}$ & 187 & 200 & $\frac{100}{60}$ \\
\hline 37 & 64 & 32 & 138 & 70 & & $\begin{array}{l}81 \\
88\end{array}$ & $\begin{array}{r}0 \\
14\end{array}$ & $\frac{0}{7}$ & $\frac{188}{189}$ & $\frac{150}{80}$ & $\frac{60}{40}$ \\
\hline 38 & 81 & 41 & 139 & 13 & $\frac{5}{7}$ & 89 & 12 & 6 & 190 & 35 & $\frac{40}{17}$ \\
\hline 39 & 47 & 23 & 140 & 63 & 32 & 90 & 81 & 41 & 191 & 0 & 0 \\
\hline 40 & 38 & 19 & 141 & 25 & 13 & 91 & 77 & 39 & 192 & 0 & 0 \\
\hline 41 & 150 & 120 & 142 & 55 & 28 & 92 & 5 & 2 & 193 & 0 & 0 \\
\hline 42 & 81 & 41 & 143 & 34 & 17 & 93 & 43 & 22 & 194 & 0 & 0 \\
\hline 43 & 81 & 41 & 144 & 52 & 26 & 94 & 32 & 16 & 195 & 0 & 0 \\
\hline 44 & 81 & 41 & 145 & 81 & 41 & 95 & 11 & 5 & 196 & 0 & 0 \\
\hline 45 & 58 & 29 & 146 & 68 & 34 & $\frac{96}{97}$ & 52 & 26 & 197 & 0 & 0 \\
\hline 46 & 52 & 26 & 147 & 40 & 20 & 98 & $\frac{19}{47}$ & $\frac{40}{24}$ & $\frac{198}{199}$ & 0 & 0 \\
\hline 47 & 0 & 0 & 148 & 52 & 26 & $\begin{array}{l}99 \\
\end{array}$ & 52 & 26 & 200 & 0 & 0 \\
\hline 48 & 81 & 41 & 149 & 130 & 65 & 100 & 130 & 65 & 201 & 0 & 0 \\
\hline 49 & 81 & 41 & 150 & 37 & 19 & 101 & 81 & 41 & 202 & 0 & 0 \\
\hline
\end{tabular}


Tabla 7. Mejores respuestas para el sistema de prueba 1.

\begin{tabular}{|c|c|c|c|}
\hline Respuesta & $\begin{array}{c}\text { Pérdidas } \\
{[\mathbf{U S} \mathbf{\text { ] }}}\end{array}$ & $\begin{array}{c}\text { Conductores } \\
{[\mathbf{U S} \mathbf{\text { \$ }}}\end{array}$ & $\begin{array}{c}\text { Total } \\
{[\mathbf{U S} \mathbf{\text { ] }}}\end{array}$ \\
\hline 1 & $222.045,34$ & $125.436,09$ & $347.481,44$ \\
\hline 2 & $211.263,24$ & $138.192,94$ & $349.456,18$ \\
\hline 3 & $234.619,43$ & $116.506,30$ & $351.125,73$ \\
\hline 4 & $216.324,91$ & $134.365,88$ & $350.690,79$ \\
\hline 5 & $216.514,82$ & $134.365,88$ & $350.880,70$ \\
\hline 6 & $227.296,93$ & $121.609,04$ & $348.905,97$ \\
\hline 7 & $220.001,80$ & $131.293,07$ & $351.294,87$ \\
\hline 8 & $211.100,71$ & $138.192,94$ & $349.293,65$ \\
\hline 9 & $214.907,99$ & $134.747,66$ & $349.655,65$ \\
\hline Referencia [4] & $208.313,60$ & $143.081,51$ & $351.395,11$ \\
\hline
\end{tabular}

correspondientes a la inversión en calibres de conductores. En esta solución debido a la baja demanda de los nodos existen únicamente tres tramos de red con calibre tipo 2 y el resto del sistema con calibre tipo 1 .

Con respecto a la regulación de tensión, para este sistema de prueba se obtiene una regulación de tensión para todos los nodos inferior al $3,85 \%$, es decir, que todos los nodos se encuentran dentro de los límites operativos establecidos.

\section{Resultados $\boldsymbol{E}_{2}$}

La mejor solución encontrada para el sistema de prueba 1 en el escenario de variación de demanda fue de US\$206.134,06 equivalente a unas pérdidas de US\$107.254,54 con un costo de inversión de US\$ 98.879,51. El nodo con mayor caída de tensión registró una regulación del 2,15\%. Los calibres asociados a la mejor solución encontrada, se presentan en la columna $\boldsymbol{E}_{\mathbf{2}}$ de la Tabla 4.

La mejor solución encontrada para el sistema de prueba 2 en el escenario de variación de demanda fue de US\$ 633.518,67 equivalente a unas pérdidas de US\$196.139,38 con un costo de inversión de US\$ 437.379,29. El nodo con mayor caída de tensión registró una regulación del 4,49\%.

\section{Análisis comparativo}

Al observar las diferencias en términos de función objetivo que presentan los escenarios de demanda $\boldsymbol{E}_{1}$ y $\boldsymbol{E}_{2}$, se nota que influye considerablemente en la componente de la función objetivo asociada a la inversión en conductores, debido a que unas pérdidas anuales divididas en tres niveles asociados a máxima, media y mínima demanda, implican que una combinación más económica de calibres de conductores, lo que permite minimizar los costos de inversión y operación del sistema.

Las variaciones porcentuales entre ambos escenarios de demanda (reducción de $\boldsymbol{E}_{2}$ con respecto a $\boldsymbol{E}_{1}$ ) corresponde al $40,68 \%$ y $31,47 \%$, para los sistemas de prueba 1 y 2 , respectivamente. Estas reducciones tan significativas en la función objetivo implican que las DisCo deben tener en cuenta al momento de realizar la expansión de sus redes de distribución, información confiable de la demanda, ya que grandes diferencias entre el valor proyectado y el valor real pueden causar sobrecostos en la inversión y, por tanto, pérdidas económicas notables para la DisCo.

Tabla 8. Comparación del tipo de calibre empleado, según el escenario de demanda.

\begin{tabular}{|c|c|c|c|c|}
\hline \multirow{2}{*}{ Calibre } & \multicolumn{2}{|c|}{ Sistema de prueba 1 } & \multicolumn{2}{c|}{ Sistema de prueba 2 } \\
\cline { 2 - 5 } & $\mathbf{E}_{\mathbf{1}}$ & $\mathbf{E}_{\mathbf{2}}$ & $\mathbf{E}_{\mathbf{1}}$ & $\mathbf{E}_{\mathbf{2}}$ \\
\hline 1 & 1 & 1 & 145 & 145 \\
\hline 2 & 0 & 2 & 15 & 23 \\
\hline 3 & 0 & 2 & 10 & 12 \\
\hline 4 & 3 & 1 & 10 & 3 \\
\hline 5 & 1 & 0 & 7 & 5 \\
\hline 6 & 1 & 1 & 0 & 0 \\
\hline 7 & 0 & 0 & 3 & 2 \\
\hline 8 & 0 & 0 & 0 & 0 \\
\hline Costo [US\$] & $125.436,09$ & $98.879,51$ & $511.210,21$ & $437.379,29$ \\
\hline
\end{tabular}


Según lo anterior, en la Tabla 8 se muestran las diferencias en el tipo de calibre según el sistema de prueba y el escenario analizado y la variación en los costos de inversión para cada caso.

\section{CONCLUSIONES}

Se desarrolló una metodología eficiente que permite la selección óptima de los calibres de los conductores para SD con una o varias fuentes, por medio de la implementación de la técnica metaheurística de optimización BT, con la que se alcanzaron soluciones de mejor calidad que las reportadas en la literatura especializada.

Se comprobó que la implementación de una técnica de optimización metaheurística, depende ampliamente del conocimiento que posea el investigador sobre el problema y del objetivo que se trace, razón por la que se requiere de procesos de parametrización heurística y de múltiples simulaciones para poder encontrar soluciones de buena calidad, que en algunos casos pueden corresponder a la solución óptima del problema.

Se propone para las DisCo, una herramienta de utilidad para proyectar la expansión de sus redes, principalmente a mediano y corto plazo, con el fin de que pueda ser atendida de forma eficiente y oportuna toda su demanda, además de que es útil para cumplir las políticas regulatorias vigentes relacionadas con reducción de pérdidas técnicas.

El modelo presentado en este trabajo queda como un punto de partida para futuras investigaciones en las que se consideren redes de distribución trifásicas, con presencia de generación distribuida y escenarios de generación y demanda para períodos de 24 horas, con el fin de que se puedan tener costos más cercanos a la realidad, en términos de inversión y operación para los SD.

Los resultados obtenidos, en términos de pérdidas técnicas, corresponden en realidad a un indicador operativo del sistema, y aunque su cálculo es exacto en función de los datos de demanda considerados, las DisCo no pueden esperar reducciones tan significativas en las pérdidas técnicas, debido a que la demanda es una variable estocástica y sus valores pico se presentan en intervalos muy breves de tiempo. Por lo anterior, para obtener un valor más cercano a la realidad, se requiere el uso de curvas de carga discretizadas en períodos de 24 horas y para varios días de la semana.

\section{AGRADECIMIENTOS}

Al programa de Becas Doctorados Nacionales del Departamento Administrativo de Ciencia, Tecnología e Innovación de Colombia (COLCIENCIAS), según convocatoria 727 de 2015 y al programa de Doctorado en Ingeniería de la Universidad Tecnológica de Pereira.

\section{REFERENCIAS}

[1] O.D. Montoya-Giraldo, "Planeación óptima de sistemas de distribución considerando reconectadores automáticos para aislamiento de fallas y transferencia de carga", Master's thesis, Universidad Tecnológica de Pereira. Feb. 2014.

[2] A. Grajales-Otalvaro, "Algoritmo búsqueda tabú aplicado al problema de la planeación de sistemas de distribución considerando ubicación y dimensionamiento de condensadores y reguladores de tensión", Master's thesis, Universidad Tecnológica de Pereira, Augu. 2014.

[3] M. Lavorato, J.F. Franco, M.J. Rider and R. Romero. "Imposing Radiality Constraints in Distribution System Optimization Problems". In IEEE Transactions on Power Systems. Vol. $27 \mathrm{~N}^{\circ}$ 1, pp. 172-180. Feb. 2012. DOI: 10.1109/TPWRS.2011.2161349

[4] J. Castilho-Neto and A.M. Cossi. "Alocação de cabos em redes de distribuição de energia elétrica de média tensão (mt) utilizando algoritmo Chu \& Beasley". In Simpósio Brasileiro de Sistemas Eletricos (SBSE). Foz do Iguaçu, Brasil. Apr. 2014.

[5] O.D. Montoya, A. Grajales, R.A. Hincapié, M. Granada and R.A. Gallego. "Methodology for optimal distribution system planning considering automatic reclosers to improve reliability indices". In Transmission \& Distribution Conference and Exposition Latin America (PES T\&D-LA). Medellín. Colombia. Sept. 2014. DOI: 10.1109/ TDC-LA.2014.6955232

[6] Zhuding Wang, Haijun Liu, D.C. Yu, Xiaohui Wang and Hongquan Song. "A practical 
approach to the conductor size selection in planning radial distribution systems". In IEEE Transactions on Power Delivery. Vol. $15 \mathrm{~N}^{\circ}$ 1, pp. 350-354, Jan. 2000. DOI: $10.1109 / 61.847272$

[7] H. Falaghi, M. Ramezani, M.R. Haghifam and K.R. Milani, "Optimal selection of conductors in radial distribution systems with time varying load". $18^{\text {th }}$ International Conference and Exhibition Electricity Distribution. Turin, Italy, 2005. DOI: 10.1049/ cp: 20051351

[8] F. Mendoza, D. Requena, J.L. Bemal-Agustin and J.A. Domínguez-Navarro. "Optimal Conductor Size Selection in Radial Power Distribution Systems Using Evolutionary Strategies". In IEEE/PES Transmission \& Distribution Conference and Exposition: Latin America. Caracas. Venezuela. 2006. DOI: 10.1109/TDCLA.2006.311451

[9] S. Sivanagaraju, N. Sreenivasulu, M. Vijayakumar and T. Ramana. "Optimal conductor selection for radial distribution systems". In Electric Power Systems Research. Vol. $63 \mathrm{~N}^{\circ} 28$, pp. 95-103, Sept. 2002.

[10] Damanjeet Kaur and Jaydev Sharma. "Optimal conductor sizing in radial distribution systems planning". In International Journal of Electrical Power \& Energy Systems. Vol. 30. No 4, pp. 261-271. May 2008.

[11] J.F. Franco, M.J. Rider, M. Lavorato and R. Romero. "Optimal Conductor Size Selection and Reconductoring in Radial Distribution Systems Using a Mixed-Integer LP Approach". In IEEE Transactions on Power Systems. Vol. $28 \mathrm{~N}^{\circ} 1$, pp. 10-20. Feb. 2013. DOI: 10.1109/TPWRS.2012.2201263
[12] G. Bakkabulindi, M.R. Hesamzadeh, M. Amelin and I.P. Da Silva. "Models for conductor size selection in Single Wire Earth Return distribution networks". In AFRICON. Port Louis. Mauricio. 2013. DOI: 10.1109/ AFRCON.2013.6757773

[13] F. Glover. "Tabu search fundamentals and uses", pp. 1-85. Fecha. 16/01/207 URL: http://leeds-faculty.colorado.edu/glover/ TS\%20-\%20Fundamentals\%26Uses.pdf

[14] B.M. Batista and F. Glover, "Introducción a la búsqueda tabú", pp. 1-36. Fecha. 16/01/207 URL: http://leeds-faculty.colorado. edu/glover/fred \%20pubs/329\%20-\%20 Introduccion $\% 20 a \% 201 a \% 20$ Busqueda $\% 20$ Tabu\%20TS_Spanish\%20w\%20Belen (119-06).pdf

[15] D. Shirmohammadi, H.W. Hong, A. Semlyen and G.X. Luo. "A compensation-based power flow method for weakly meshed distribution and transmission networks". In IEEE Transactions on Power Systems. Vol. $3 \mathrm{~N}^{\circ}$ 2, pp. 753-762. May. 1988. DOI: $10.1109 / 59.192932$

[16] A.M. Eldurssi and R.M. O'Connell. "A Fast Nondominated Sorting Guided Genetic Algorithm for Multi-Objective Power Distribution System Reconfiguration Problem". In IEEE Transactions on Power Systems. Vol. 30 N $^{\circ}$ 2, pp. 593-601. March. 2015. DOI: 10.1109/TPWRS.2014.2332953

[17] O.D. Montoya, R.A. Hincapié and M. Granada. "Nuevo enfoque para la localización óptima de reconectadores en sistemas de distribución considerando la calidad del servicio y los costos de inversión". In Ingeniare. Revista chilena de ingeniería. Vol. $24 \mathrm{~N}^{\circ}$ 1, Jan. 2016, pp. 55-69. 